\title{
Bacterionomics and vironomics in carcinogenesis
}

\author{
Pratiwi Sudarmono* \\ Department of Microbiology, Faculty of Medicine Universitas Indonesia, Jakarta, \\ Indonesia
}

DOI: http://dx.doi.org/10.19106/JMedScieSup004804201626

\section{ABSTRACT}

Virus and bacteria are microbes which are the most common cause human infection. Most of the bacterial infection can be eradicated by antibiotics and infection symptoms disappear. However, eradication is more complicated in viral infection, because viruses will persistently stay in the host, some even undergoes not only lytic cycle but also integration into host genome. Nowadays, there are at least 6 virus types consistently related to human cancer, such as EBV, HPV, HTLV, HBV, HCV, HKSV, and the new one Merkel Virus (MCV). Although not every infected people will get cancer, around $20 \%$ of the whole cancers in human are caused by viral oncogene.

Class one oncogenic bacteria is Helicobacter pylori. Infection with this bacterium causes persistent gastro-duodenal inflammation, which in turn alters gastric cell growth and transformation. Expression of Cag gene and Vac gene and some expression of OMP protein are usually linked to gastric cancer.

The complex molecular mechanisms of carcinogenesis for every infective virus include several processes triggered by cell transformation. In addition, other host and environmental factors play significant roles in cancer development. Some scientist put a hallmark analysis as a model to quickly summarize the ongoing pathobiological process willtand which gene or protein caused the process. The hallmark analysis is comprised of several process which may happen simultaneously, because some of the hallmark is caused by the same protein. The hallmark consists of various virus strategies in oncogenesis such as promoting angiogenesis, avoiding immune destruction, triggering genome instability and mutation, deregulating cellular energetics, resisting cell death, sustaining proliferative signals, evading growth suppressors, enabling cellular immortality, promoting inflammation, and triggering metastasis. For example, HPV infection will cause low-grade dysplasia which might continue to become invasive cervical cancer. After host cell transformation, in the long control region genes, E6 and E7 protein will promote several strategies in oncogenesis including resisting cell death and evading growth suppressors. HBV infection will end without any serious liver damage, but after cell transformation, almost all hallmark strategies of viral oncogenesis are happening step by step in line with the severity of hepatocellular damage.

As the onset of cancer development after infection can last for years, there is an opportunity to design either vaccine or genetic therapy to minimize further risk of human cancers.

Keywords: oncogenic bacterial, viral, oncogenesis, suppressor, oncogene

Corresponding author: psdrmn@yahoo.com 